\author{
CARLES SANCHIS IBOR ${ }^{a}$ \\ RUTGERD BOELENS ${ }^{\mathrm{b}}$ \\ MARTA GARCÍA-MOLLÁc
}

\title{
CAMBIO TECNOLÓGICO, PRIVATIZACIÓN Y RECUPERACIÓN DE LA AUTOGESTIÓN COMUNITARIA DEL RIEGO. EL CASO DE SENYERA (VALÈNCIA)
}

\begin{abstract}
RESUMEN
Los procesos de cambio de tecnología del riego están facilitando la penetración silenciosa de la empresa privada en los sistemas de gestión colectiva del riego en numerosos lugares de España y otros países mediterráneos. Este artículo analiza este fenómeno a partir del caso de estudio de Senyera (València), siguiendo la privatización, la disputa y la posterior recolectivización de la gestión por parte de los usuarios del riego. El trabajo muestra como la privatización menoscaba la autonomía de los agricultores y eleva los costes de gestión en un contexto de escasa transparencia. También revela la capacidad de los regantes para reelaborar su pasada acción colectiva, dando lugar a una nueva forma de autogestión creativa.

Palabras clave: Acción colectiva; privatización del agua; gestión del riego; economía moral del agua; riego por goteo.
\end{abstract}

\section{Abstract}

\section{TECHNOLOGICAL CHANGE, PRIVATIZATION AND RECOVERY OF COMMUNITY SELF-MANAGEMENT OF IRRIGATION. THE CASE OF SENYERA (VALÈNCIA)}

Processes of technological change foster the silent penetration of private enterprise in collective irrigation water management in numerous areas of Spain, and in other Mediterranean countries. This

a Centro Valenciano de Estudios del Riego, Universitat Politècnica de València, Camí de Vera s/n, 46022 València. csanchis@hma.upv.es

b Department of Environmental Sciences, Wageningen University, The Netherlands. rutgerd.boelens@wur.nl CEDLA Centre for Latin American Research and Documentation, The Netherlands.

Department of Geography, Planning and International Development Studies, University of Amsterdam, The Netherlands.

c Centro Valenciano de Estudios del Riego, Universitat Politècnica de València, Camí de Vera s/n, 46022 València. mgarmo@esp.upv.es

Fecha de recepción: septiembre 2018. Fecha de aceptación: noviembre 2018. 
paper discusses this phenomenon through a case study of the community of Senyera (València), tracking the privatization and subsequent contestation and re-takeover of water management by irrigation system users. The article shows how privatization removes users' autonomy and increases irrigation costs in a context of little transparency. The case also highlights users' capacity to re-value and re-signify their past collective action, turning it into new, creative forms of self-governance.

Keywords: Collective action; water privatization; irrigation management; moral economy of water; drip irrigation.

\section{Neoliberalismo, tecnología de Riego y economía moral del agua}

Desde la década de 1980, llevada por el auge del neoliberalismo, el agua ha estado sujeta a procesos de mercantilización y privatización en todo el mundo (Harvey, 2003; Bakker, 2005; Swyngedouw, 2005; Castro, 2007). La privatización de los servicios públicos de suministro de agua potable ha sido el fenómeno más frecuente, e indudablemente el más contestado. De hecho, se observa una creciente remunicipalización del agua en ciudades de diferentes tamaños en todo el mundo (Pigeon et al., 2012; Lobina et al., 2015). Para resituarse en este contexto, los proveedores privados están buscando nuevos nichos de mercado (Bakker, 2013; Yacoub et al., 2015), tratando de posicionarse en otros sectores como la gestión del riego, hasta ahora ajenos a la intervención de las empresas privadas.

Más allá de políticas institucionales mercantilizadoras y privatizadoras del agua, las empresas privadas suelen aprovecharse de los procesos de modernización tecnológica/tecnicista, para penetrar y privatizar los bastiones de gestión colectiva del agua. Este es un fenómeno incipiente y apenas estudiado en España. Por lo general, las empresas privadas aprovechan los proyectos de construcción, como la creación de nuevas áreas regables, de nuevas infraestructuras de suministro o la transformación en riego presurizado. Las empresas responsables de introducir las nuevas infraestructuras de riego alcanzan acuerdos con las juntas de gobierno de las entidades de riego y/o la administración pública, a veces incorporando el servicio de mantenimiento y gestión a los contratos de construcción, otras veces contratándolo después. De esta manera, pasan a controlar el sistema de riego inmediatamente después de inaugurarlo. Es una fórmula que se produce silenciosa y sutilmente en entidades de riego de diferentes regiones españolas (Sanchis-Ibor et al., 2017a).

Hay distintas modalidades para fomentar la penetración de la empresa privada. En algunos casos, las instituciones no pierden el control de sus funciones tradicionales, aunque incorporan fórmulas de contratación de servicios y conocimiento externo. Sin embargo, en otras ocasiones se ha detectado una transferencia total de la gestión comunitaria del riego a empresas. En estos casos, las antiguas prácticas que sostenían los valores culturales y morales esenciales para la coexistencia de las comunidades rurales han sido reemplazadas por métodos de gestión empresarial que siguen criterios de eficiencia y búsqueda de beneficios. Es una sutil transformación sociotécnica que apenas ha recibido atención hasta la fecha. 
Mediante la modernización tecnológica por «expertos», la penetración de la empresa privada abre una brecha en los sistemas de gestión colectiva. Esto ocurre también en aquellos sistemas de riego autogestionados que, como instituciones que han perdurado en el tiempo, han demostrado una gran sostenibilidad, robustez y resiliencia (Ostrom, 1990; Hidalgo et al., 2017; Mena et al., 2017). Se basan en marcos normativos y derechos de agua colectivos ligados a valores sociales, culturales y morales que son diferentes de los marcos y las prácticas de gestión neoliberales (Vos et al., 2006; Vos and Boelens, 2014; Roth et al., 2015). Su naturaleza los ha hecho tradicionalmente inaccesibles o poco atractivos para las empresas privadas y las agencias gubernamentales, precisamente porque trascienden los parámetros económicos y burocráticos convencionales (Cleaver, 2000; Hoogesteger, 2012; Roa García, 2014).

Los colectivos que gestionan sus sistemas de riego son entidades socialmente diversas; no caben análisis romantizados ni presentaciones utópicas o idealizadas, ya que se producen en contextos de relaciones de poder («externos» e «internos») a menudo muy desiguales. Sin embargo, es importante considerar que estos sistemas están constituidos por miembros unidos por una dependencia mutua: para desarrollar, usar y administrar sus recursos hídricos, mediante un sentido de identidad colectiva del agua (Boelens, 2011, 2014). Los derechos y obligaciones de cada miembro se derivan de los derechos y deberes comunes, y en caso de conflicto existe un gran interés colectivo en resolverlo rápidamente, restaurar la cooperación efectiva (Garrido, 2011). Esta reciprocidad colectiva, a veces de recorrido plurisecular, difiere radicalmente de los acuerdos contractuales basados en el mercado (Reimer et al., 2008); se basa en la confianza, la moral de la comunidad, la historia compartida de la defensa del agua y la cohesión social a largo plazo. Esta naturaleza de la gestión colectiva del agua ha sido destacada por varios autores como una derivación de la teoría de la economía moral desarrollada por Thompson (1971) o Scott (1976) o la teoría del pluralismo legal (Benda-Beckmann et al. 1998, Roth et al., 2015). Así, por ejemplo, Ferri (1997) y González Alcantud (1998) han explorado los principios normativos o morales que tradicionalmente regían el manejo del agua de riego en Valencia y Andalucía; Gelles (2000), Trawick (2001) y Boelens $(2014,2015)$ han descifrado críticamente tales visiones en los Andes; Arellano (2014) en Nuevo México; y Cleaver $(2000,2017)$ y ElDidi y Corbera (2017) en diferentes lugares de África.

La dimensión moral del riego se materializa cuando se crean infraestructuras y normas para la acción colectiva. Tanto la tecnología como las reglas de gestión son plasmaciones de principios éticos y estructuras sociales que definen cómo se comparten cargas, riesgos y beneficios (Meehan, 2013; Boelens y Vos, 2014). En consecuencia, los procesos de cambio tecnológico reflejan también cambios en las concepciones y la moralidad del agua, que en ocasiones pueden ser ajenas a estas colectividades, cuando la intervención del Estado o de otros agentes, al modificar las infraestructuras de las comunidades, puede imponer visiones que difieren de las tradiciones locales. Esta misma razón es por la cual, cuando se producen 
estos cambios tecnológicos, se abren las puertas a la empresa privada, y esta flanquea una muralla de saberes tradicionales para tomar el control de la plaza.

\section{Metodología y caso de estudio}

Hemos estudiado este proceso en Senyera (València), un caso que presenta un interés destacado debido a que permite analizar el tránsito desde la gestión comunitaria hasta la privada, la gestión privatizada durante 10 años, y luego la vuelta a la «gestión recolectivizada», tras la recuperación del control del sistema de riego comunitario por parte de los agricultores. Senyera es un pueblo valenciano de 1.169 habitantes ubicado cerca de la confluencia de los ríos Júcar y Albaida. Desde la época medieval, los campos de Senyera han sido regados con agua del río Albaida a través de la Séquia Comuna d’Ènova. Este canal se divide en tres brazos, uno de los cuales, la Séquia del Terç, abastece los campos de Senyera — actualmente 116 ha- y de Castelló de la Ribera. Todos los regantes de Senyera son miembros del Sindicat de Regs, una asociación para la gestión colectiva del riego y la defensa de sus intereses comunes. Las explotaciones (inferiores a media hectárea de promedio) se han dedicado en los últimos 20 años casi exclusivamente al cultivo del naranjo; los agricultores tienen una media de edad avanzada (casi la mitad tienen más de 65 años) y predomina el trabajo a tiempo parcial. El área cultivada era de 140 ha a principios de este siglo, pero disminuyó a 116 ha (387 parcelas) como resultado de los proyectos de desarrollo urbano. Actualmente solo están en riego 77,5 ha (283 parcelas) (Jiménez-Bello et al., 2007, 2010).

La información se recopiló a través de entrevistas semiestructuradas de entre una hora y dos horas y media de duración, realizadas en enero y junio de 2014 , junio y diciembre de 2015 y junio de 2016 (antes, durante y después de la recolectivización), con los miembros anteriores y actuales de la junta directiva de la entidad de riego; técnicos anteriores y actuales de asociaciones de usuarios, la empresa privada (Tecvasa); los tres últimos alcaldes de Senyera; agricultores locales y de las comunidades de regantes vecinas. Las entrevistas de invierno se celebraron en la sede de la entidad de riegos y se centraron en la recopilación de información económica y técnica del sistema de riego, de antes, durante y después de la privatización. Las entrevistas de verano se realizaron junto a tres grupos de 4-5 estudiantes cotutelados por los autores, tanto en los bares que servían de lugar de reunión a los regantes, como en las oficinas de Tecvasa y el Ayuntamiento de Senyera. Sus materiales se presentaron en tres informes inéditos (De Beer et al., 2014; Führen et al., 2015; Borghuis, et al., 2016). Algunos de los actores clave fueron entrevistados varias veces al año, y se llevaron a cabo reuniones de grupos de interés y entrevistas colectivas con algunos de ellos, así como visitas conjuntas a las instalaciones de riego de la entidad. No se emplearon sistemas de grabación para no cohibir a los entrevistados. 


\section{SeNYeRA: gestión COLECTIVA, PRIVATIZACión Y RECOleCtiviZACIÓN}

\subsection{El riego a manta en una pequeña comunidad citrícola}

Cuando recuerdan el periodo de control comunitario del agua, «antes de que llegara la empresa», los regantes idealizan su modelo de gestión, elevándolo a la categoría de elemento identitario y factor de cohesión interna. El reparto del agua seguía un ritual que articulaba buena parte de la vida diaria de la comunidad. «Aunque las personas tienen diferentes recuerdos de los hechos, miran hacia atrás como un sistema equitativo» (Borghuis et al., 2016: 18).

El regador contratado por la comunidad organizaba turnos de riego generales y los agricultores podían efectuar pequeñas adaptaciones aunque, en general, el que llevaba esperando el agua más tiempo la recibía primero. En verano, la transparencia en la gestión del agua la garantizaba la presencia del regador en el bar El Moreno. Estar allí a unas horas determinadas todos los días hacía la gestión accesible. En invierno o en años lluviosos, el tablón de anuncios del bar publicaba el cronograma y no era necesaria la intervención del regador. Los agricultores regaban a demanda, después de recoger un palo (el palet) y depositar tres euros al dueño del bar, lo que certificaba el turno de riego. Los agricultores tenían que plantar el palet en su campo y cuando terminaban, el siguiente agricultor que quería regar lo recogía y le pagaba el depósito. La información compartida rápidamente entre los agricultores, la negociación fluida, la socialización y la transparencia de la programación del riego contribuían a satisfacer a los usuarios: los agricultores sabían cuándo esperar su agua, incluso durante las sequías.

La toma de decisiones informal tenía lugar en los bares, ya que eran (y siguen siendo) el centro social del pueblo. Un lugar de reunión todas las mañanas para el esmorzar, donde comían y bebían juntos, un lugar en el que se discutía el estado de los campos, el agua y cualquier necesidad y problema que tuvieran. Las cuestiones debatidas en el bar se traducían en propuestas para las reuniones de la asamblea general o la junta de gobierno, para su posterior formalización (Führen, et al., 2015). Las obligaciones del usuario, como el trabajo colectivo o las tarifas del agua ( $180 €$ por hectárea/año) se percibían como justas, ya que todos podían pagarlas, sabían por qué las estaban pagando y eran conscientes que la comunidad de regantes no pretendía buscar ganancias. En general, según los entrevistados, el riego era una parte apreciada de la vida pública diaria.

La infraestructura de agua de Senyera y su racionalidad reflejaban no solo las circunstancias agroclimáticas y geofísicas de la región, sino también las fuerzas sociales, culturales y políticas dominantes en la comunidad. Durante las entrevistas, los agricultores de Senyera y sus familias expresaron, de formas diversas, como su sistema de gestión colectiva reafirmaba un sentimiento de pertenencia a la comunidad, establecido a través del riego, dando lugar a una identidad y una cultura hidráulica. 


\subsection{Riego por goteo y privatización}

Esta cultura del agua fue desarticulada en 2004, cuando el Sindicat recibió una propuesta del Ayuntamiento de Senyera, firmemente interesado en transformar por completo la histórica red de riego en un moderno sistema de riego por goteo. El proyecto fue presentado ante la Asamblea General para su aprobación, y el alcalde del pueblo y un comercial de una empresa de riegos intervinieron desde la mesa presidencial para explicar y defender el proyecto de transformación. La opinión general de los agricultores entrevistados es que fueron mal informados, manipulados y persuadidos por el poder discursivo de la modernización y la privatización. A la asamblea comunitaria asistieron solo 55 de los 235 miembros, que aprobaron el proyecto con 47 votos a favor. De las cinco ofertas presentadas por diferentes compañías para ejecutar la transformación, eligieron Tecvasa, el más caro pero el único que ofrecía financiación. Algunos regantes discreparon del plan de Tecvasa. Como expresó un ex presidente de la comunidad: «El nuevo alcalde no quería las ideas de nadie más. [...] A cualquier precio, debería instalarse riego por goteo [...] El "quórum" era abiertamente ilegal, pero logró comenzar su proyecto por un llamado "ingeniero" que no era independiente, y la compañía recibió el encargo por más de 1 millón de euros».

Una vez que la asamblea estuvo de acuerdo, la compañía presionó para que los trámites se hicieran rápidamente y, de hecho, en unos pocos días se firmó el contrato, incluida la asignación completa de la administración del sistema durante 10 años. Las obras se iniciaron rápidamente. Según los regantes entrevistados, Tecvasa, que también asumió el control y la gestión del suministro de agua potable del municipio, trató a los agricultores con poca transparencia. Algunos regantes entendieron que la compañía contribuiría con el 50 \% de los costos, pero en realidad este montante provenía de las subvenciones del gobierno regional para modernizar el riego. Los representantes de la junta de gobierno de 2004 también nos hablaron de sus dificultades para tratar de comprender la redacción legal del contrato. Como afirma uno de los miembros de aquella junta: «El contrato estaba escrito de manera que era imposible de entender. Me senté para leerlo con el diccionario, pero una palabra me llevaba a otra, y así sucesivamente, hasta volver a la primera de nuevo. Nunca entendí lo que significaba». Otros entrevistados afirmaron que el documento que Tecvasa presentó en la asamblea comunitaria era sustancialmente diferente del contrato enviado posteriormente para la firma. El contrato firmado con la empresa los comprometía con un pago obligatorio y forzaba a los regantes a indemnizar a la empresa si decidían abandonar el proyecto. Esta cláusula no dejó espacio para reconsiderar la posición de la comunidad.

A lo largo de 2004, se instaló el riego por goteo y el nuevo sistema comenzó a funcionar. La mayoría (pero no todos) los agricultores cambiaron al riego por goteo en sus campos. De todos los agricultores consultados ninguno indicó el ahorro de agua como un incentivo para el cambio, aunque este discurso político es el que había justificado la modernización del riego por goteo. Valoraban esencialmente la comodidad del riego: se evitaba el riego nocturno, se 
reducía el tiempo de dedicación y se obtenía una mayor facilidad para agregar fertilizantes. El nuevo sistema incorporaba un embalse de $98.000 \mathrm{~m}^{3}$ para almacenar agua del río, y una red de distribución que disponía de 52 hidrantes de múltiples salidas y un total de 331 tomas. La infraestructura pronto reveló deficiencias operativas debido a un diseño y construcción inadecuados. La ubicación del depósito y las bombas elegidas para presurizar la red —bombas sumergible - fueron una opción errónea, por su baja eficiencia energética. Además, la red estaba sobredimensionada, como sucede a menudo en proyectos de modernización de riego. Para hacerlos «rentables» (Boelens, 2015; Sanchis-Ibor et al., 2016, 2017a, 2017b) se suele basar el diseño del sistema en un área mayor de la realmente regada, lo que permite presentar un menor costo por hectárea y facilitar la aprobación, pese a que redunda en costos de construcción más altos de lo necesario.

\subsection{Descontento y reacción comunitaria}

En 2006, una nueva junta directiva se hizo cargo de la comunidad de regantes y solicitó a la Asociación Valenciana de Agricultores un informe para evaluar la posible revocación de los acuerdos con Tecvasa. El documento decepcionó a los regantes, pues confirmó que no tenían forma legal de recuperar la gestión del riego hasta que el contrato expirara en 10 años.

La frustración de los regantes fue creciendo a medida que surgían problemas de diseño y gestión de la red. Los defectos de diseño de la red de presión provocaron numerosas rupturas de las conducciones. Además, los regantes comenzaron a tener problemas con algunos cultivos. El riego por goteo no era efectivo para los árboles más viejos, porque no cubría toda su área radicular, afectando a la producción.

El desencanto de los regantes también creció cuando vieron cómo perdían la autonomía y la transparencia en la administración de la entidad. Tecvasa designó a varios operarios para gestionar la infraestructura, siguiendo un programa de riego informatizado desarrollado por un equipo de expertos universitarios. No había contacto directo entre los operarios del sistema y los agricultores con respecto a la asignación de agua, lo que dificultó que los agricultores organizaran, negociaran y adaptaran los turnos diarios de riego a sus necesidades. Diferentes culturas chocaron, como expresó un agricultor: «Tuve un conflicto con un ingeniero de Tecvasa y dije: sabes mucho sobre libros, pero no sobre el campo», mientras que Tecvasa culpaba a la comunidad por no funcionar de acuerdo con los estándares: «La comunidad necesita desarrollarse profesionalmente, pero existen fuerzas sociales y políticas que lo inhiben» (Borghuis et al., 2016: 16)

El Sindicat perdió la mayor parte de su autoridad operativa, ya que Tecvasa estaba a cargo de todas las decisiones de gestión. El presidente actual observa: «Constantemente teníamos que recordarle a Tecvasa que nosotros éramos los propietarios, que ellos solo eran proveedores de servicios, pero no nos escucharon ni nos entendieron». Tecvasa también impedía a los agricultores ver los contadores de riego, cerrados en cajas metálicas, para comprobar 
si el volumen de agua que llegaba a sus campos coincidía con la factura de la empresa. La imposibilidad de controlar los contadores de riego obligó a la junta de gobierno a examinar detalladamente la facturación, y comenzaron a detectarse numerosas facturas con errores, siempre a favor de la compañía.

El aumento de los costes del riego irritó sobremanera a los regantes. Los costes de operación y mantenimiento se triplicaron, de $180 € /$ ha a $572 € /$ ha. Además de estos costos, pagaron $4.918 € /$ ha distribuidos en diez anualidades para amortizar la obra. El costo total de riego, en consecuencia, se multiplicó por seis en los 10 años que Tecvasa administró el sistema de riego, alcanzando $1.064 € /$ ha. La compañía también obtuvo grandes beneficios al introducir la fertirrigación centralizada. Los precios de los fertilizantes, raramente más de 400 $€ /$ ha antes de la transformación, alcanzaron $1.680 € /$ ha en las parcelas con riego por goteo.

Para la mayoría de los pequeños agricultores, vivir bajo administración privatizada fue duro. El contexto de bajos precios de naranja, unido a los altos costos de riego gestionado por Tecvasa dejó poco margen de beneficio. Un agricultor con una parcela promedio de 0,5 ha apenas ganaba $600 €$ al año, sin contar los costos de mano de obra y pesticidas, por lo que en algunos casos apenas dejaba margen para pagar por recoger las naranjas. El contrato firmado con Tecvasa también fue especialmente costoso para la comunidad cuando los usuarios incurrían en cualquier morosidad. Si algún regante no pagaba a tiempo, era el Sindicato en su conjunto el que tenía que cubrir el descubierto y pagar a la compañía directamente. La compañía también impuso una cláusula antiabandono, por lo que los agricultores tenían que pagar incluso si renunciaban a sus cultivos y derechos de agua.

A pesar de los grandes problemas con la gestión y el servicio prestado, Tecvasa trató de promover este caso como ejemplar, colaborando con varios investigadores universitarios. Se realizaron estudios sobre el riego deficitario (González-Altozano, 2007), se diseñó un SIG para integrar la agronomía y el agua para gestionar las comunidades de regantes (Jiménez-Bello et al., 2007); se realizaron varias evaluaciones técnicas para aumentar la eficiencia en la distribución de fertilizantes (Jiménez-Bello et al., 2010); y se desarrollaron mejoras para minimizar el consumo de energía (Jiménez-Bello et al., 2011). Sin embargo, los técnicos que trabajaron para Tecvasa afirmaron que las mejoras conseguidas en los costes de riego de este trabajo, debidas a la reducción de los costos de energía, no se repercutieron a los regantes. Solo sirvieron para aumentar los márgenes de beneficio de la empresa.

La compañía también se benefició al reducir el mantenimiento del sistema de riego al mínimo. Según los representantes del Sindicat, la empresa no contrató ningún seguro para la infraestructura y descuidó las instalaciones, especialmente en los últimos años, de modo que dejaron varios elementos fuera de servicio cuando el contrato terminó. Al acercarse el final del contrato de 10 años, la opinión general de los agricultores fue que «la operación técnica y el mantenimiento se pueden hacer mucho más baratos, y debe hacerlo alguien con una pasión agrícola, de la propia comunidad» (De Beer et al., 2014: 21). Por el contrario, Tecvasa era un firme creyente en el síndrome de los expertos, quería continuar y asumió que 
había hecho que los agricultores dependieran suficientemente de sus servicios expertos y afirmaron estar «seguros de que será difícil para los usuarios del agua administrar el goteo por sí mismos» (De Beer et al., 2014: 21).

Fundamentalmente, Tecvasa siguió la moralidad del mercado. Al contrario del control colectivo del agua y la moralidad de los medios de subsistencia de los agricultores de Senyera: «... para Tecvasa, la implementación del goteo en Senyera fue un caso de prueba. Ejecutó el proyecto para expandir los negocios en el riego. Aprovecharon las oportunidades en Senyera para involucrarse en el riego por goteo debido a los subsidios disponibles» (De Beer at al. 2014: 24), y para ver cuánto beneficio podían sacar de la comunidad de pequeños agricultores.

Durante 10 años, los agricultores perdieron margen de beneficio, transparencia y autonomía. La insatisfacción con la gerencia de la compañía desanimó a los regantes de asistir a asambleas generales. Tecvasa solo aparecía ocasionalmente en estas reuniones. Según los representantes de la empresa, se negaron a asistir a las asambleas generales porque consideraban que era « $\ldots$ una democracia falsa: de entre todos los miembros, solo hay unos pocos que asisten» (citado en Borghuis et al. 2016: 21). Sin embargo, en la última asamblea general del decenio de gestión de Tecvasa, celebrada para decidir sobre la posible renovación del contrato, la asistencia fue masiva.

\subsection{El retorno a la gestión colectiva}

Finalmente, a principios de 2015, cuando expiró el contrato, la comunidad decidió retomar el control del sistema de riego. Aunque Tecvasa quería continuar con su trabajo, el presidente actual explica: «Nos negamos. ¡Estábamos hartos de ellos!». El Sindicat contrató a un técnico local para administrar el sistema de riego y comenzó a tomar diferentes medidas para reducir los costes de riego. Los resultados del primer año de gestión colectiva fueron muy positivos, un tremendo alivio económico para los agricultores de Senyera. La comunidad ha reducido los costes de gestión del riego a $468 € /$ ha, un descenso del $18,1 \%$. Por su parte, los costes de fertirrigación disminuyeron en un $67,8 \%$ (de $1.680 € /$ ha a $541 € /$ ha) gracias a las acciones de la junta directiva. Al mismo tiempo, los agricultores dicen que han observado una mejora sustancial en las condiciones de sus árboles con los nuevos fertilizantes: «los árboles están verdes ahora», afirmó un miembro de la comunidad. Y a pesar de la reducción sustancial de los costes, el Sindicato ha finalizado los años fiscales de 2015 y 2016 con un superávit en sus cuentas. En la misma línea, como concluyeron Borghuis et al. (2016: 25) «Aunque generalmente se cree que el mercado abierto abarata los servicios, la autogestión comunitaria de Senyera muestra lo contrario», ya que los beneficios obtenidos de la gestión redundan directamente en el beneficio de los usuarios individuales.

Entre los regantes que resistieron la privatización había partidarios y detractores del riego por goteo. Pero una vez instalado el sistema y recuperado el control de la comunidad, fue 
fácil establecer la cohabitación entre ambos grupos. El sistema de riego de Senyera ahora se basa en dos tipos de riego: tanto por gravedad como a presión. Para riego por inundación de los campos, un canal secundario proporciona el agua que llega a través de pequeñas compuertas o paletes. Junto con el técnico local de goteo, se ha recuperado la figura del regador, que controla el sistema de riego por gravedad. Como Borghuis et al. (2016: 14) explican: «Los agricultores se ponen en contacto con él para solicitar un turno de riego, para lo cual el agricultor puede elegir ordenar abrir y cerrar la compuerta o hacerlo por sí mismo. Los agricultores que usan riego por goteo también tienen acceso al riego a manta, que utilizan durante los períodos cálidos». Tanto la balsa como el pozo comunitario (solo se usa en caso de sequía) se comparten para poder proporcionar agua al riego a manta y al goteo.

Los usuarios de agua de Senyera demuestran que la tecnología de riego no es un elemento autónomo que puede dictar los patrones de la vida social y cultural. El uso, manejo y resultados de los sistemas de riego pueden ser redefinido; sus scripts originales «no proporcionan la única manera de hacer el trabajo» (Pfaffenberger, 1992: 498). Los agricultores querían más flexibilidad en la conducción del agua y artefactos de aplicación, y han adaptado el hardware a sus necesidades, instalándole un nuevo software.

Además, los agricultores entrevistados expresan una gran satisfacción por la recuperación de la gestión colectiva de su riego: «Ahora lo gestionamos nosotros. Estamos orgullosos de tener el sistema en nuestras manos». Cuando el sistema automático de riego por goteo falla, el operador del sistema controla manualmente la asignación del agua y los agricultores le hablan directamente si desean cambios particulares (por ejemplo, un ciclo de riego adicional). Se ha restablecido el contacto cara a cara (o teléfono directo) con el operador del sistema, y este disfruta de confianza y cercanía similar a los antiguos regadores. Los agricultores expresan su fe tanto en la ejecución de las tareas de control de agua como en el pago de sus cuotas, ya que ahora pueden leer fácilmente en las facturas las cantidades que les han cobrado: «Ahora tenemos más trabajo, pero es mucho mejor. Ahora trabajamos para nosotros mismos. Ahora el operador trabaja para nosotros y nos da toda la información». Este no fue el caso durante la etapa de privatización. Curiosamente, cuando la junta de gobierno del Sindicat sugirió que se redujera el salario del técnico respecto a lo que cobraba el empleado de Tecvasa, los agricultores se opusieron a esto, enfatizando la buena relación entre el operador del sistema y los usuarios. "No es solo una cuestión de dinero; también es una cuestión de orgullo» (agricultor citado en Führen, et al., 2015: 16).

La recolectivización también ha revitalizado las reuniones en el bar, tanto informales como formales, ordinarias y extraordinarias. Discutir sus modos de gobernanza del agua, derechos de agua y prácticas de asignación e infraestructura, muestra que la esencia del sistema está la autonomía en la toma de decisiones, la transparencia y la cercanía, obligaciones y precios justos, y uso y operación flexible de la tecnología. Los usuarios sienten que pueden desarrollar y practicar sus propias normas, que han sido capaces de remoralizar su tecnología y su gestión. Esto ha restaurado el clima de confianza que se había roto al privatizar la administración. 


\section{Discusión y CONCLUSIONES}

La reforma de la tecnología de riego inevitablemente rediseña la dimensión social del riego. Aunque ni los «diseñadores expertos» ni los «usuarios» son bloques morales y normativos monolíticos con intereses y suposiciones estándares — sino redes complejas que a menudo acomodan valores, normas y visiones no alineados-, los cambios en la infraestructura afectan a las normas y organizaciones y, por lo tanto, puede alterar sus valores intangibles.

Cuando estos cambios suceden dentro de los organismos de gestión colectiva, por decisión propia y bajo el control autónomo de los usuarios, se produce una adaptación bidireccional interactiva. Por un lado, la comunidad aplica o modifica la tecnología de acuerdo con sus criterios morales y adapta la nueva infraestructura a sus necesidades, interviniendo o supervisando el diseño de ingeniería para reformar el sistema de riego, o más tarde, introduciendo ajustes y creando sus propios protocolos. Por otro lado, a la inversa, si es impuesta sutil o verticalmente, la tecnología adoptada puede inducir cambios significativos en la estructura y dinámica de la comunidad, a menudo inesperadamente, lo que obliga a la entidad a reorganizarse para ajustarse o aprovechar las demandas y el potencial del nuevo hardware.

El caso de Senyera podría haber encajado en estos parámetros, ya que la mayoría de los agricultores no tenían objeciones significativas para implementar la nueva tecnología, pese a que no compartían los objetivos generalmente asociados con esta tecnología de riego («optimización de la eficiencia»). En lugar de ahorrar agua o aumentar la producción, los agricultores de Senyera, en su mayoría de edad avanzada, querían la comodidad que ofrece esta tecnología, principalmente para dejar de tener que regar durante la noche. Sin embargo, en Senyera, la nueva tecnología se asimiló mediante un proceso conflictivo. En primer lugar, el cambio de tecnología y gestión no fue por iniciativa de la comunidad, sino que fue propuesto por agentes externos, con objetivos y moralidad completamente ajenos a la entidad de agricultores colectivos. Además, los regantes percibieron el cambio tecnológico como invasivo, ya que estuvo acompañado por la gestión privatizadora, la desposesión de algunos derechos fundamentales y la ruptura de sus prácticas diarias, hechos que impidieron cualquier una adaptación satisfactoria.

El caso de Senyera, como otros analizados en España (Ortega-Reig et al., 2017), Marruecos (Houdret, 2012; Bossenbroek, 2016), Egipto (ElDidi y Corbera, 2017), India (Birkenholtz, 2016), los países andinos (Boelens y Gelles, 2005; Roa García, 2014; Paerregaard et al., 2016; Boelens et al., 2015) y en muchos otros países del mundo (Bakker, 2013; Jackson, 2018; Wilson y Inkster, 2018), pone de relieve el contraste entre la lógica de la gestión colectiva del riego y la de las empresas privadas. Ambas sirven a objetivos dispares y por tanto abocan en una desigual socialización de los beneficios derivados de la gestión. La privatización de la gestión externalizó y expropió temporalmente el patrimonio social y material de los regantes de Senyera. Este proceso es más amplio que la expropiación económica: no solo expropia parte de los productos del proceso de riego y los medios de producción, sino tam- 
bién las definiciones locales de lugar de trabajo, tiempo de trabajo y ritmo de trabajo. Así, en Senyera, las habilidades y el conocimiento local respecto al diseño y uso del agua fueron presa de este proceso expropiador.

Como muestra este caso, la dependencia de las comunidades frente al control político-operativo «exterior» se define no solo por disposiciones legales y estructuras organizativas (neoliberales y/o modernizadoras) sino también por los detalles del diseño técnico de riego. En Senyera, el diseño de la red impidió que los usuarios vieran los contadores de riego, oscureciendo la transparencia del sistema, rompiendo los lazos de larga duración entre los regantes, y entre estos y el regador, y de ese modo las bases para la confianza de los usuarios del sistema. Este análisis es de crucial importancia, porque a pesar del hecho que muchas organizaciones políticas de influencia internacional (como las Naciones Unidas; véase Shah et al., 2018) han tomado cierta distancia del «Consenso de Washington» (que aboga por la privatización de la gestión de los recursos naturales), las mismas siguen cerrando sus ojos por los impactos negativos de la «privatización mediante la modernización tecnológica» en la gestión del agua. El mito del progreso a través de la modernización universalista mediante tecnologías de riego «avanzadas» tiene rasgos casi dogmáticos —en España y en el mundo (Pfaffenberger, 1988; Vos y Boelens, 2014; Sanchis-Ibor et al., 2017a; Roth et al., 2015; Venot et al., 2017).

En Senyera, Tecvasa también se quedó con el derecho de decidir sobre los horarios, la distribución del agua y la elección y cantidad de fertilizante en el agua. Los agricultores mantuvieron sus derechos de acceso al agua pero perdieron su derecho a usar y controlar la infraestructura, ya que no tenían acceso a las instalaciones de riego por goteo. La gestión privada, impulsada por el criterio de maximizar los beneficios, transfirió altos costos operativos a los regantes, mantuvo el mantenimiento de las inversiones al mínimo y no generó beneficios para los usuarios de las mejoras tecnológicas que fueron gradualmente incorporadas en el sistema de riego. Incluso desde una perspectiva exclusivamente económica, contrariamente a los discursos neoliberales sobre la gestión privada, la experiencia de Senyera muestra cómo la acción colectiva administra los bienes comunales de manera más eficiente que la gestión de la empresa privada. Gran parte de esta ventaja se debe precisamente a la moral comunitaria y la búsqueda del bienestar común, que la administración privatizada no puede proporcionar, porque necesita maximizar el beneficio. Esto se debe, en última instancia, a una idiosincrasia que la empresa privada nunca entendió.

El contrato no se prorrogó por varias buenas razones: altos costos operativos, falta de transparencia y acceso a las instalaciones, y disputas sobre legitimidad. Además, hubo un fuerte empuje para volver a la autonomía comunal, a través de un proceso de remembranza. Remembranza en el sentido de rememorar la gestión perdida, pero también remembranza que aquí, en cierta manera, adquiere también el sentido de volver a ser miembros de una comunidad. Los agricultores recordaron y reelaboraron sus prácticas sociales, sus redes informales y los beneficios de la acción colectiva. Todavía estaban orgullosos de su identidad, 
más unidos quizás ahora por el efecto que produce la llegada de un agente externo que toma el control de su sistema de riego.

Los agricultores se reorganizaron activando y renovando su red de gestión, recuperando las normas sociales no escritas y logrando un nivel de participación sin precedentes en las asambleas generales. También se reorganizaron a sí mismos haciendo suya la tecnología de goteo, adaptando el hardware a sus necesidades y combinando el riego localizado con inundaciones periódicas a través de la antigua red de gravedad. Todo esto dio como resultado un proceso de regeneración, recobrando la autonomía, el autogobierno y la acción colectiva. El sistema de riego ha sido remoralizado de acuerdo con los antiguos principios y prácticas que gobiernan los bienes comunes en la región desde la época medieval, pero asimilando el nuevo hardware.

\section{ReFERENCIAS}

Arellano, J.E. (2014): Enduring Acequias. Wisdom of the land, knowledge of the water. University Press, Alburquerque, New Mexico.

BAKKeR, K. (2005): Neoliberalizing Nature? Market environmentalism in water supply in England and Wales. Ann. Ass. Amer. Geogr., 95(3), 542-565.

Bakker, K. (2013): Neoliberal versus postneoliberal water: geographies of privatization and resistance. Ann. Assoc. Am. Geogr. 103 (2), 253-260.

Beer de, P., Bosma, L., Rouw, M. y Hagenvoort, J. (2014): Effects of Modernisation in a Traditional Irrigation System - The Case of Senyera. Wageningen University, Wageningen.

Benda-Beckmann, F. von, Benda-Beckmann K. von y Spiertz J. (1998): Equity and legal pluralism: taking customary law into account in natural resource policies, in: R. Boelens y G. DÁvila (eds): Searching for Equity, pp. 57-69, Van Gorcum, Assen.

Birkenholtz, T. (2016): Dispossessing irrigators: water grabbing, supply-side growth and farmer resistance in India. Geoforum, 69, 94-105.

Boelens, R. (2011): Luchas y defensas escondidas. pluralismo legal y cultural como una práctica de resistencia activa y creativa en la gestión local del agua en los Andes. Anuario de Estudios Americanos, 68(2), 673-703.

Boelens, R. (2014): Cultural politics and the hydrosocial cycle: Water, power and identity in the Andean highlands. Geoforum, 57, 234-247.

Boelens, R. (2015): Water, Power and Identity. The Cultural Politics of Water in the Andes. Routledge/ Earthscan, London and Washington DC.

Boelens, R., y Gelles P.H. (2005): Cultural Politics, Communal Resistance and Identity in Andean Irrigation Development. Bulletin of Latin American Research, 24(3), 311-327.

Boelens, R. y Vos, J. (2014): Legal Pluralism, Hydraulic Property Creation and Sustainability: The materialized nature of water rights in user-managed systems. Current Opinion in Environmental Sustainability, COSUST, 11, 55-62. 
Boelens, R, J. Hoogesteger y M. Baud (2015): Water reform governmentality in Ecuador: neoliberalism, centralization and the restraining of polycentric authority and community rule-making. Geoforum, 64:281-291, doi: 10.1016/j.geoforum.2013.07.005

Borghuis, G., M.de Boer, A. Prieto, P. van Leeuwen y K. van Krieken (2016): Equity changes due to irrigation modernization. Senyera, Spain. Wageningen University, Wageningen.

Bossenbroek, L. (2016): Behind the Veil of Agricultural Modernization: Gendered Dynamics of Rural Change in the Saïss, Morocco. Wageningen University, Wageningen.

Castro, J.E. (2007): Poverty and citizenship: sociological perspectives on water services and publicprivate participation. Geoforum, 38(5), 756-771.

Cleaver, F. (2000): Moral Ecological Rationality, Institutions and the Management of Common Property Resources. Development and Change, 31(2), 361-383

Cleaver, F. (2017): Everyday water injustice and the politics of accommodation. In: R. Boelens, T. Perreault, J. Vos, M. Zwarteveen. Water Justice, pp. 246-258. Cambridge University Press, Cambridge.

ElDidi H. y Corbera E. (2017): A moral economy of water: charity wells in Egypt's Nile Delta. Development and Change. Development and Change, 48(1), 121-145.

FERri, M. (1997): Reorganización de los regadíos valencianos en el siglo XIX: Las ordenanzas liberales de la provincia de Valencia (1835-1850). Areas: Revista Internacional de Ciencias Sociales, 17, 77-90.

Führen, H., Kolkman, I., Mirshadiev, M. y van Spronsen, A. (2015): Oranges, Money $\mathcal{G}$ Autonomy - An investigation of the irrigation management transfer in Senyera, Valencia. Wageningen University, Wageningen.

Garrido, S. (2011): Las instituciones de riego en España del Este. Una reflexión a la luz de la obra de Elinor Ostrom. Historia Agraria, 53, 13-42.

Gelles, P.H. (2000): Water and Power in Highland Peru: The Cultural Politics of Irrigation and Development. Rutgers University Press, New Brunswick, NJ.

González Alcantud, J.A. (1998): Economía moral del agua. Demófilo, 27, 199-207.

GonzÁlez-Altozano, P. (2007): Estudio de demostración, en campos convencionales de Navelina, de la aplicación de estrategias de riego deficitario controlado. Research Report, Valencian Institute of Agrarian Research (IVIA), Generalitat Valenciana.

Harvey, D. (2003): The New Imperialism. Oxford University Press.

Hidalgo, J.P., R. Boelens y Vos J. (2017): De-colonizing water. Dispossession, water insecurity, and Indigenous claims for resources, authority, and territory. Water History, 9, 67-85. DOI 10.1007/ s12685-016-0186-6.

Hoogesteger, J. (2012): Democratizing Water Governance from the Grassroots: The Development of Interjuntas-Chimborazo in the Ecuadorian Andes. Human Organization, 71(1), 76-86.

Houdret, A. (2012): The water connection: Irrigation and politics in southern Morocco. Water Alternatives, 5(2), 284-303.

JACKSON S. (2018): Water and Indigenous rights: Mechanisms and pathways of recognition, representation, and redistribution. WIREs Water 5, online, doi: 10.1002/wat2.1314. 
Jiménez-Bello, M.A., Martínez-Alzamora, F., Arviza, J., Manzano, J., Ríos, N. y Aguilella, J. (2007): Herramientas para el uso racional del agua en sistemas de riego a presión con el apoyo de un GIS (Huragis). Riegos y Drenajes XXI, 152, 54-61.

Jiménez-Bello, M.A., Martínez-Alzamora, F., Bou, V. y Bartolín, H. (2010): Analysis, assessment, and improvement of fertilizer distribution in pressure irrigation systems. Irrigation Science, 29, 45-53.

Jiménez-Bello, M.A., Martínez-Alzamora, F., Castel, J.R. y Intrigliolo, D.S. (2011): Validation of a methodology for grouping intakes of pressurized irrigation networks into sectors to minimize energy consumption. Agricultural Water Management, 102, 46-53.

Lobina, E., Kishimoto, S. y Petitjean, O. (2015): Here to Stay: Water Remunicipalisation as a Global Trend. Transnational Institute, London.

Meenan, K. (2013): Disciplining de facto development: water theft and hydrosocial order in Tijuana. Environment and Planning, 31, 319-336.

Mena-VÁsconez, P., L. Vincent, J. Vos y R. Boelens (2017): Fighting over water values: diverse framings of flower and food production with communal irrigation in the Ecuadorian Andes. Water International, 42(4), 443-461, DOI 10.1080/02508060.2017.1309511

Ortega-Reig, M., Sanchis-Ibor, C., Palau-Salvador, G., García-Mollá, M., y Avellá-Reus, L. (2017): Institutional and management implications of drip irrigation introduction in collective irrigation systems in Spain. Agricultural Water Management, 187, 164-172, DOI: 10.1016/j. agwat.2017.03.009

Ostrom, E. (1990): Governing the commons: the evolution of institutions for collective action. Cambridge University Press, Cambridge, UK.

Paerregatrd, K., Bredholt Stensrud, y A. Oberborbeck Andersen, A. (2016): Water Citizenship: Negotiating Water Rights and Contesting Water Culture in the Peruvian Andes. Latin American Research Review, 51(1), 198-217.

Pfaffenderger, B. (1992): Social anthropology of technology. Annu. Rev. Anthropol. 21:491-516.

Pigeon, M., McDonald, D.A., Hoedeman, O. y Kishimoto, S. (Eds.) (2012): Re-Municipalisation: Putting Water Back into Public Hands. Transnational Institute, Amsterdam.

Reimer, B., Lyons, T., Ferguson, N. y Polanco, G. (2008): Social Capital as Social Relations: The Contribution of Normative Structures. Sociological Review, 56(2), 256-274, doi: 10.1111/j.1467954X.2008.00787.x.

Roa García, M.C. (2014): Equity, efficiency and sustainability in water allocation in the Andes: Trade-offs in a full world. Water Alternatives, 7(2), 298-319.

Roth, D., R. Boelens y Zwarteveen, M. (2015): Property, legal pluralism, and water rights: the critical analysis of water governance and the politics of recognizing «local» rights. Journal of Legal Pluralism and Unofficial Law, 47(3), 456-475.

Sanchis-Ibor, C., García-Mollá, M. y Avellà, L. (2016): Efectos de la modernización del regadío en las entidades de riego de la Comunidad Valenciana, Boletín de la Asociación de Geógrafos Espanoles, 72, 9-36. 
Sanchis-Ibor, C., Boelens, R. y García-Mollá. M. (2017a): Collective irrigation reloaded. Re-collection and re-moralization of water management after privatization in Spain. Geoforum, 87, 3847, DOI: 10.1016/j.geoforum.2017.10.002.

Sanchis-Ibor, C.; García-Mollá, M. y Avellà, L. (2017b): Effects of drip irrigation promotion policies on water use and irrigation costs in Valencia, Spain. Water Policy, 19(1), 165-180, DOI: 10.2166/wp.2016.025.

Scotr, J.C. (1976): The Moral Economy of the Peasant: Rebellion and Subsistence in Southeast Asia. Yale University Press, New Haven.

Shah, E., J.W. Liebrand, J. Vos, G. J. Veldwisch y R. Boelens (2018): The UN World Water Development Report 2016, Water and Jobs: A Critical Review. Development and Change, 49(2), 678 691, doi: 10.1111/dech.12395.

Swyngedouw, E. (2005): Dispossessing $\mathrm{H}_{2} \mathrm{O}$ : the contested terrain of water privatization. Capital. Nat. Social, 16(1), 81-98.

Thompson, E.P. (1971): The Moral Economy of the English Crowd in the Eighteenth Century. Past and Present, 50(1), 76-136.

Trawick, P. (2001): The moral economy of water: equity and antiquity in the Andean commons. American Anthropologist, 103, 361-379.

Venot J.P., M. Kuper y M. Zwarteveen (Eds.): 2017. Drip Irrigation: Untold Stories of Efficiency, Innovation $\mathbb{E}$ Development. Earthscan/Routledge, London

Vos, H. de, Boelens, R. y Bustamante, R. (2006): Formal Law and Local Water Control in the Andean Region: A Fiercely Contested Field. International Journal of Water Resources Development, 22(1), 37-48, doi: 10.1080/07900620500405049.

Vos, J. y Boelens, R. (2014): Sustainability Standards and the Water Question. Development and Change, 45(2), 205-230, doi: 10.1111/dech.12083.

Wilson. N.J. y InKsTeR, J. (2018): Respecting water: Indigenous water governance, ontologies and the politics of kinship on the ground. Environment and Planning E: Nature and Space, online: https:// doi.org/10.1177/2514848618789378

Yacoub, C., Duarte, B. y Boelens, R. (Eds.) (2015): Agua y Ecología Política. El extractivismo en la agro-exportación, la minería y las hidroeléctricas en Latino América. Abya-Yala, Quito.

Cómo citar este artículo:

Sanchis Ibor, C., Boelens, R. y García-Mollá, M. (2018). Cambio tecnológico, privatización y recuperación de la autogestión comunitaria del riego.

El caso de Senyera (València). Cuadernos de Geografía, 101, 91-106.

https://doi.org/10.7203/CGUV.101.13722

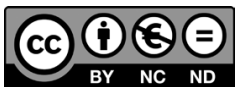

Este obra está bajo una licencia de Creative Commons Reconocimiento-NoComercial-SinObraDerivada 4.0 Internacional. 